\title{
Massive MIMO TWO-Hop Relay Systems Over Rician Fading Channels
}

\author{
Jian Cao*1, Shujuan $\mathbf{Y u}^{2}$, Jie Yang ${ }^{3}$, Yun Zhang ${ }^{4}$, Shengmei Zhao ${ }^{5}$ \\ ${ }^{1}$ Nanjing University of Posts and Telecommunications \\ Nanjing, 210023 -China \\ [e-mail: 18260039728@163.com, yusj@njupt.edu.cn, 1017020718@njupt.edu.cn,y021001@njupt.edu.cn, \\ Zhaosm@njupt.edu.cn] \\ *Corresponding author: Jian Cao
}

Received March 9, 2019; revised May 30, 2019; accepted June 8, 2019;

published November 30, 2019

\begin{abstract}
With the advent of the fifth-generation (5G) era, Massive multiple-input multiple-output (MIMO) relay systems have experienced the rapid development. Recently, the performance analysis models of Massive MIMO relay systems have been proposed, which are mostly based on Rayleigh fading channels. In order to create a more suitable model for 5G Internet of Things scenarios, our study is based on the Rician fading channels, where line-of-sight (LOS) path exists in the channels. In this paper, we assume the channel state information (CSI) is perfect. In this case, we use statistical information to derive the analytical exact closed-form expression for the achievable sum rate of the uplink for the Massive MIMO two-hop relay system over Rician fading channels. Moreover, considering the different communication scenarios, we derive the analytical exact closed-form expression for the achievable sum rates of the uplink for other three scenarios. Finally, based on these expressions, we make simulations and analyze the performance under different transmit powers and Rician-factors, which provides a theoretical basis and reference for further research.
\end{abstract}

Keywords: Massive MIMO, two-hop relay, Rician fading channel, Rician-factor, LOS path

This research was supported by National Natural Science Foundation of China (No.61302155, No.61274080, No. 61871234); This work was also supported by National Natural Science Fund Incubation Project of China (NY214052). 


\section{Introduction}

As an essential part of the 5G wireless communication field, the Massive MIMO system has aroused wide concern of researchers [1-3]. In order to improve the quality of service (QoS) and increase coverage of MIMO systems, MIMO relay systems have also attracted great attention in recent years [4-9]. The main disadvantage of the Massive MIMO relay systems is the serious path loss since there is no direct link between the user and the destination base (BS), and the signal transmitted by the user must be relayed by relay (RS) in order to finally reach BS. Both RS and BS are equipped with a large number of antenna arrays, which provides unprecedented spatial freedom to serve multiple users terminals simultaneously on the same time-frequency channel. Under the premise of perfect CSI and being available at the RS and BS, maximum ratio combining (MRC) with noise reduction and interference can increase both the spectral and energy efficiency [10-12].

In recent years, amplify-and-forward (AF) relay technology has attracted the attention of researchers. It not only improves the quality of QoS of cell edge users, which effectively increases coverage, but also provides reliable links for areas with severe path loss [13-15]. For example, with the application of AF technology, multipair Massive MIMO one-way relay has been studied in [13], where the received signals are processed respectively by MRC and zero-forcing (ZF) in the relay. Under the premise of adequate relay antennas, [13] derives the approximate closed-form expression of the achievable rate with MRC compared to ZF processing when the transmission power is reduced to the $1 / \mathrm{N}$ (the number of relay antennas) of original. What's more, in order to expand the coverage area of wireless communication, multipair Massive MIMO two-way relay has been studied in [14]. They derive the closed-form expression of the achievable rate with the assumption that the number of relay antennas is large enough and approaches infinity. The simulation results show that, the rate of the analytical approximate result is infinitely close to the rate of the simulation result with the increasing number of antennas. In [15], multipair Massive MIMO two-way relay networks were proposed to improve the QoS of wireless communication and further expand convergence. However, the previous work on AF relay systems was mainly based on Rayleigh fading channels, which ignored the effect of LOS component in realistic wireless communication scenarios. Therefore, it is of great significance to research the MIMO relay system over Rician fading channel.

In this paper, we focus on a Massive MIMO two-hop relay system featured with LOS path over Rician fading channels, which is different from traditional work. The main contributions of this paper are as follows: Firstly, through the simple process of received signals with the usage of MRC at RS and the application of AF technology, we derive the

analytical exact closed-form expression for the achievable sum rate of the uplink using 
statistical information. Then, based on the presence or absence of LOS path from user to RS and RS to BS, we develop three analytical exact closed-form expressions for the achievable sum rates of the uplink under three different scenarios. In detailed, the first case is based on the presence of LOS path between the user and RS. Similarly, the second case is based on the presence of LOS path between the user and BS. And the third case is based on the absence of LOS path between user to RS or RS to BS). Finally, with the foundation of above expressions, we conduct simulations and evaluate the performance under different transmit powers and Rician-factors.

Notations: Throughout the paper, the uppercase boldface letters represent the matrix, and the lowercase boldface letters represent the column vectors. $(.)^{H}$ and $\operatorname{tr}($.$) denote the$ conjugate transpose and trace of the matrix, respectively. The complex Gaussian distribution with * mean and variance $\sigma^{2}$ is expressed as $\mathcal{C N}\left(*, \sigma^{2}\right) . E\{$.$\} represent the$ expectation. $\mathbf{I}_{N}$ denotes an $N \times N$ identify matrix. And $[\mathbf{X}]_{i j}$ or $\mathbf{X}_{i j}$ gives the $(i, j)$ th entry of $\mathbf{X}$.

\section{System Model}

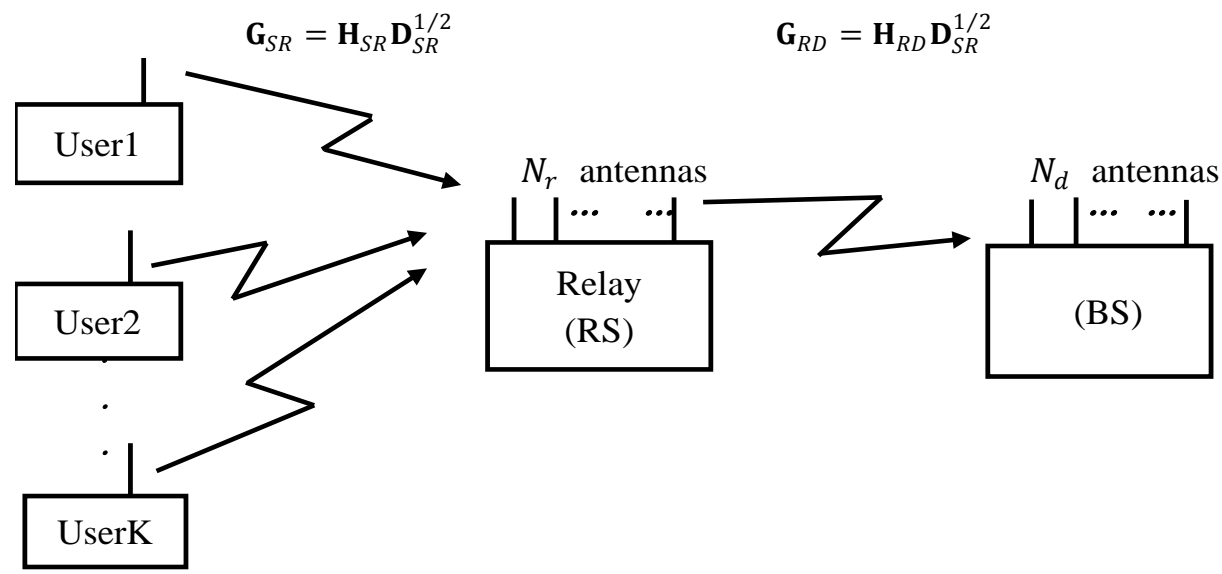

Fig. 1. System model of a Massive MIMO two-hop relay uplink

Fig. 1 displays an uplink model of the Massive MIMO relay system over the Rician fading channel, which includes RS with $N_{r}$ antennas, BS with $N_{d}$ antennas and $K$ single-antenna users. The signal transmitted by the $K$ user must be relayed by the relay RS in order to finally reach BS. The channel between the user to RS $\mathbf{G}_{S R}$, similarly, the channel between RS and BS is denoted as $\mathbf{G}_{R D}$. And there is LOS path both in $\mathbf{G}_{S R}$ and $\mathbf{G}_{R D}$. Besides, there are $N_{r}$ and $N_{d}$ antennas respectively in the relay RS and BS, which satisfy $N_{d} \geq N_{r}>K$. 


\subsection{Channel Model}

The user to RS and the RS to BS channel coefficients can be expressed as $g_{S R, m k}=$ $\left[\mathbf{G}_{S R}\right]_{m k}, g_{R D, n k}=\left[\mathbf{G}_{R D}\right]_{n k}$, which can be written as

$$
\begin{aligned}
& g_{S R, m k}=h_{S R, m k} \sqrt{\alpha_{k}} \\
& g_{R D, n k}=h_{R D, n k} \sqrt{\beta_{k}}
\end{aligned}
$$

Where $h_{S R, m k}$ is a fast fading element between the $k$ th user and the $m$ th antenna of the RS, and $h_{R D, n k}$ is a fast fading element between the $k$ th optional transmit antennas of the RS and the $n$th antenna of the BS. Besides, $\alpha_{k}$ and $\beta_{k}$ are the large-scale fading coefficients [16]. Matrix form can be expressed as

$$
\begin{aligned}
& \mathbf{G}_{S R}=\mathbf{H}_{S R} \mathbf{D}_{S R}^{1 / 2} \\
& \mathbf{G}_{R D}=\mathbf{H}_{R D} \mathbf{D}_{R D}^{\mathbf{1 / 2}}
\end{aligned}
$$

Where $\mathbf{D}_{S R}$ and $\mathbf{D}_{R D}$ are the $K \times K$ diagonal matrix with $\left[\mathbf{D}_{S R}\right]_{k k}=\alpha_{k}$ and $\left[\mathbf{D}_{R D}\right]_{k k}=\beta_{k} \cdot \mathbf{H}_{S R}$ and $\mathbf{H}_{R D}$ denote $N_{r} \times K$ and $N_{d} \times K$ fast fading matrices from the user to the RS and the RS to the BS, i.e., $\left[\mathbf{H}_{S R}\right]_{m k}=h_{S R, m k}$ and $\left[\mathbf{H}_{R D}\right]_{n k}=$ $h_{R D, n k}$. The fast fading matrices $\mathbf{H}_{S R}$ and $\mathbf{H}_{R D}$ consist of deterministic component $\overline{\mathbf{H}}_{S R}$, $\overline{\mathbf{H}}_{R D}$ and Rayleigh distribution random component $\mathbf{H}_{S R, w}, \mathbf{H}_{R D, w}$, respectively. Then, the matrices $\mathbf{H}_{S R}$ and $\mathbf{H}_{R D}$ can be expressed as [17]

$$
\begin{aligned}
\mathbf{H}_{S R} & =\overline{\mathbf{H}}_{S R}\left[\boldsymbol{\Omega}_{S R}\left(\boldsymbol{\Omega}_{S R}+\mathbf{I}_{K}\right)^{-1}\right]^{1 / 2}+\mathbf{H}_{S R, w}\left[\left(\boldsymbol{\Omega}_{S R}+\mathbf{I}_{K}\right)^{-1}\right]^{1 / 2} \\
\mathbf{H}_{R D} & =\overline{\mathbf{H}}_{R D}\left[\boldsymbol{\Omega}_{R D}\left(\boldsymbol{\Omega}_{R D}+\mathbf{I}_{K}\right)^{-1}\right]^{1 / 2}+\mathbf{H}_{R D, w}\left[\left(\boldsymbol{\Omega}_{R D}+\mathbf{I}_{K}\right)^{-1}\right]^{1 / 2}
\end{aligned}
$$

Where both $\boldsymbol{\Omega}_{S R}$ and $\boldsymbol{\Omega}_{R D}$ represent the a Rician-factor diagonal matrix of $K \times K$, and the Rician-factor of the $k$ th user can represent $\left[\boldsymbol{\Omega}_{S R}\right]_{k k}=\mu_{k}$ and $\left[\boldsymbol{\Omega}_{R D}\right]_{k k}=\varepsilon_{k}$, respectively. The entries of $\mathbf{H}_{S R, w}$ and $\mathbf{H}_{R D, w}$ are Gaussian random variables with zero mean and variance of $1 / 2$, independent and identical distribution. $\overline{\mathbf{H}}_{S R}$ and $\overline{\mathbf{H}}_{R D}$ can be written as [18-20]

$$
\begin{aligned}
{\left[\overline{\mathbf{H}}_{S R}\right]_{m k} } & =e^{-j(m-1)\left(2 \pi d_{S R} / \lambda\right) \sin \left(\theta_{S R, k}\right)} \\
{\left[\overline{\mathbf{H}}_{R D}\right]_{n k} } & =e^{-j(n-1)\left(2 \pi d_{R D} / \lambda\right) \sin \left(\theta_{R D, k}\right)}
\end{aligned}
$$

Where $\theta_{S R, i}$ and $\theta_{R D, i}$ represent the angle of arrival of the $i$ th user at RS and BS, respectively. $\lambda$ is the wavelength. $d_{S R}$ and $d_{R D}$ represent the spatial distances at the user to RS and RS to BS, respectively.

\subsection{Signal Processing}

Let $\mathbf{x}_{S} \in \mathbb{C}^{K \times 1}$ be the signal transmitted by $K$ single antenna users, which satisfies $\mathbb{E}\left\{\mathbf{x}_{S} \mathbf{x}_{S}{ }^{H}\right\}=\mathbf{I}_{K}$ [6]. $\mathbf{G}_{S R}$ is a channel matrix of $N_{r} \times K$ from users to RS. The received signal $\mathbf{y}_{R}$ at the RS is

$$
\mathbf{y}_{R}=\sqrt{p_{u}} \mathbf{G}_{S R} \mathbf{x}_{S}+\mathbf{n}_{R}
$$


where $p_{u}$ is the transmission power of each single antenna user and $\mathbf{n}_{R}$ is additive white Gaussian noise (AWGN) with $\mathcal{C N}\left(0, \sigma_{R}{ }^{2} \mathbf{I}_{\boldsymbol{n}_{R}}\right)$. After receiving the signal $\mathbf{y}_{R}$ at the RS, simple signal processing is performed via the MRC by multiplying the received signal vector $\mathbf{y}_{R}$ at the RS with the MRC matrix $\mathbf{W}_{S R}^{H}$ which depends on $\mathbf{G}_{S R}^{H}$. The processed signal can be expressed as

$$
\mathbf{x}_{R}=\mathbf{W}_{S R}^{H} \mathbf{y}_{R}=\sqrt{p_{u}} \mathbf{G}_{S R}^{H} \mathbf{G}_{S R} \mathbf{x}_{S}+\mathbf{G}_{S R}^{H} \mathbf{n}_{R}
$$

Then the signal is amplified and then forwarded, and the forwarded signal $\widetilde{\mathbf{x}}_{R}$ can be expressed as

$$
\tilde{\mathbf{x}}_{R}=\gamma \mathbf{x}_{R}
$$

Where $\gamma$ is the amplification factor satisfying the constraint $\mathbb{E}\left\{\tilde{\mathbf{x}}_{R}^{\mathrm{H}} \tilde{\mathbf{x}}_{R}\right\}=P_{R}$. Using $\mathbb{E}\left\{\tilde{\mathbf{x}}_{R}^{\mathrm{H}} \tilde{\mathbf{x}}_{R}\right\}=P_{R}$ and combine (10) and (11), we can obtain

$$
\gamma=\sqrt{\frac{P_{R}}{\left.p_{u} \operatorname{tr}\left(\mathbb{E}\left\{\mathbf{G}_{S R}^{H} \mathbf{G}_{S R} \mathbf{G}_{S R}^{H} \mathbf{G}_{S R}\right\}\right)+\sigma_{R}^{2} \operatorname{tr}\left(\mathbb{E}\left\{\mathbf{G}_{S R}^{H} \mathbf{G}_{S R}\right\}\right\}\right)}}
$$

Then the power amplified signal $\tilde{\mathbf{x}}_{R}$ of $K$ users is transmitted by $K$ antennas at the RS optionally. Transmitted signal $\tilde{\mathbf{x}}_{R}$ by the user passes through the Rician fading channel $\mathbf{G}_{R D}$ to the BS. The received signal $\mathbf{y}_{D}$ at the BS is

$$
\mathbf{y}_{D}=\mathbf{G}_{R D} \tilde{\mathbf{x}}_{R}+\mathbf{n}_{D}
$$

Where $\mathbf{G}_{R D}$ is the channel matrix of $N_{d} \times K$ between RS and BS, $\mathbf{n}_{D} \sim \mathcal{C N}\left(0, \sigma_{D}{ }^{2} \mathbf{I}_{n_{D}}\right)$ is the AWGN at the BS. After receiving the signal $\mathbf{y}_{D}$ at the BS, simple signal processing is performed via the MRC, that is conducted by multiplying the received signal vector $\mathbf{y}_{D}$ at the BS with the MRC matrix $\mathbf{W}_{R D}^{H}$ which depends on $\mathbf{G}_{R D}^{H}$. Finally, the received signal vector of $K$ users at the BS is

$$
\begin{gathered}
\mathbf{r}_{D}=\mathbf{W}_{R D}^{H} \mathbf{y}_{D} \\
=\gamma \sqrt{p_{u}} \mathbf{G}_{R D}^{H} \mathbf{G}_{R D} \mathbf{G}_{S R}^{H} \mathbf{G}_{S R} \mathbf{x}_{S}+\gamma \mathbf{G}_{R D}^{H} \mathbf{G}_{R D} \mathbf{G}_{S R}^{H} \boldsymbol{n}_{R}+\mathbf{G}_{R D}^{H} \boldsymbol{n}_{D}
\end{gathered}
$$

The analysis of the achievable rate for received signal will be elaborated in the next chapter.

\section{Analysis of Achievable Uplink Rate}

\subsection{Sum Rate of Massive MIMO Two-Hop Relay System over Rician Fading Channel}

Assuming that there is perfect CSI from the user to the RS and the RS to the BS, and without channel estimation. According to equation (14), the acceptance signal of the $k$ th user at the BS can be expressed as follows:

$$
\begin{gathered}
\mathrm{r}_{D, k}= \\
\underbrace{\gamma \sqrt{p_{u}} \mathbf{g}_{R D, k}^{H} \mathbf{G}_{R D} \mathbf{G}_{S R}^{H} \mathbf{g}_{S R, k} \mathbf{x}_{s, k}}_{\text {desired signal }}+\underbrace{\gamma \sqrt{p_{u}} \sum_{j \neq k}^{K} \mathbf{g}_{R D, k}^{H} \mathbf{G}_{R D} \mathbf{G}_{S R}^{H} \mathrm{~g}_{S R, j} \mathbf{x}_{s, j}}_{\text {interference }}+
\end{gathered}
$$




$$
\underbrace{\gamma \mathbf{g}_{R D, k}^{H} \mathbf{G}_{R D} \mathbf{G}_{S R}^{H} \mathbf{n}_{R}+\mathbf{g}_{R D, k}^{H} \mathbf{n}_{D}}_{\text {noise }}
$$

According to the (15), the simulation sum rate of the uplink for the $k$ th use is given:

$$
R_{k}=\frac{1}{2} \mathbb{E}\left\{\log _{2}\left(1+\frac{\gamma^{2} p_{u}\left|\mathbf{g}_{R D, k}^{H} \mathbf{G}_{R D} \mathbf{G}_{S R}^{H} \mathbf{g}_{S R, k}\right|^{2}}{\gamma^{2} p_{u} \sum_{j \neq k}^{K}\left|\mathbf{g}_{R D, k}^{H} \mathbf{G}_{R D} \mathbf{G}_{S R}^{H} \mathbf{g}_{S R, j} \mathbf{x}_{S, j}\right|^{2}+\gamma^{2} \sigma_{R}{ }^{2}\left|\mathbf{g}_{R D, k}^{H} \mathbf{G}_{R D} \mathbf{G}_{S R}^{H}\right|^{2}+\sigma_{D}{ }^{2}\left\|\mathbf{g}_{R D, k}^{H}\right\|^{2}}\right)\right\}
$$

Where the number of antennas is large enough [21, eq24], the formula (16) can be approximated as

$$
R_{k} \approx \frac{1}{2} \log _{2}\left(1+\frac{\gamma^{2} p_{u} \mathbb{E}\left\{\left|\mathbf{g}_{R D, k}^{H} \mathbf{G}_{R D} \mathbf{G}_{S R}^{H} \mathbf{g}_{S R, k}\right|^{2}\right\}}{\gamma^{2} p_{u} \sum_{j \neq k}^{K} \mathbb{E}\left\{\left|\mathbf{g}_{R D, k}^{H} \mathbf{G}_{R D} \mathbf{G}_{S R}^{H} \mathbf{g}_{S R, j}\right|^{2}\right\}+\gamma^{2} \sigma_{R}{ }^{2} \mathbb{E}\left\{\left|\mathbf{g}_{R D, k}^{H} \mathbf{G}_{R D} \mathbf{G}_{S R}^{H}\right|^{2}\right\}+\sigma_{D}{ }^{2} \mathbb{E}\left\{\left\|\mathbf{g}_{R D, k}^{H}\right\|^{2}\right\}}\right)
$$

Let $A_{k}=\gamma^{2} p_{u} \mathbb{E}\left\{\left|\mathbf{g}_{R D, k}^{H} \mathbf{G}_{R D} \mathbf{G}_{S R}^{H} \mathbf{g}_{S R, k}\right|^{2}\right\}, B_{k}=\gamma^{2} p_{u}\left\{\left|\mathbf{g}_{R D, k}^{H} \mathbf{G}_{R D} \mathbf{G}_{S R}^{H} \mathbf{g}_{S R, j}\right|^{2}\right\}$, $C_{k}=\gamma^{2} \sigma_{R}{ }^{2} \mathbb{E}\left\{\left|\mathbf{g}_{R D, k}^{H} \mathbf{G}_{R D} \mathbf{G}_{S R}^{H}\right|^{2}\right\}, D_{k}=\sigma_{D}{ }^{2} \mathbb{E}\left\{\left\|\mathbf{g}_{R D, k}^{H}\right\|^{2}\right\}$, the theorem1 can be obtained.

Theorem 1: The analytical exact sum rate of the uplink for Massive MIMO two-hop relay system over Rician fading channels can be expressed as

$$
R_{\text {sum }}=\frac{1}{2} \sum_{k=1}^{K} \log _{2}\left(1+\frac{A_{k}}{B_{k}+C_{k}+D_{k}}\right)
$$

$$
\mu_{k} \mu_{i} \Phi_{k i}^{2} / N_{r}+\mu_{k}+\mu_{i}+1
$$

Let $\Delta_{S R, k}=\frac{2 \mu_{k}+1}{\left(\mu_{k}+1\right)^{2}}, \Phi_{k i}=\frac{\sin \left(N_{r} \pi\left(\sin \theta_{S R, k}-\sin \theta_{S R, i}\right) / 2\right)}{\sin \left(\pi\left(\sin \theta_{S R, k}-\sin \theta_{S R, i}\right) / 2\right)}, Q_{k i}=\frac{}{\left(\mu_{k}+1\right)\left(\mu_{i}+1\right)}$,

$$
\varepsilon_{k} \varepsilon_{i} \phi_{k i}^{2} / N_{d}+\varepsilon_{k}+\varepsilon_{i}+1
$$

$\Delta_{R D, k}=\frac{2 \varepsilon_{k}+1}{\left(\varepsilon_{k}+1\right)^{2}}, \phi_{k i}=\frac{\sin \left(N_{d} \pi\left(\sin \theta_{R D, k}-\sin \theta_{R D, i}\right) / 2\right)}{\sin \left(\pi\left(\sin \theta_{R D, k}-\sin \theta_{R D, i}\right) / 2\right)}, R_{k i}=\frac{}{\left(\varepsilon_{k}+1\right)\left(\varepsilon_{i}+1\right)}$

By using the expressions of $\Delta_{S R, k}, Q_{k i}, \Phi_{k i}, \Delta_{R D, k}, R_{k i}, \phi_{k i}$, term $A_{k}, B_{k}, C_{k}, D_{k}$ in equation (18) can be written as

$$
\begin{gathered}
A_{k}=\gamma^{2} p_{u} \alpha_{k} \beta_{k} N_{r} N_{d}\left[\alpha_{k} \beta_{k}\left(N_{r}+\Delta_{S R, k}\right)\left(N_{d}+\Delta_{R D, k}\right)+\sum_{i \neq k}^{K} \alpha_{i} \beta_{i} Q_{i k} R_{k i}\right] \\
B_{k}=\gamma^{2} p_{u} \beta_{k} N_{r} N_{d} \sum_{j \neq k}^{K} \alpha_{j}\left[\alpha_{k} \beta_{k} Q_{k j}\left(N_{d}+\Delta_{R D, k}\right)+\sum_{i \neq k, i \neq j}^{K} \alpha_{i} \beta_{i} Q_{i j} R_{k i}+\alpha_{j} \beta_{j} R_{k j}\left(N_{r}+\right.\right. \\
\left.\left.\Delta_{S R, j}\right)\right] \\
C_{k}=\gamma^{2} \beta_{k} N_{r} N_{d} \sigma_{R}^{2}\left[\alpha_{k} \beta_{k}\left(N_{d}+\Delta_{R D, k}\right)+\sum_{i \neq k}^{K} \alpha_{i} \beta_{i} R_{k i}\right] \\
D_{k}=\sigma_{D}^{2} \beta_{k} N_{d}
\end{gathered}
$$

Proof: See Appendix A

According to the expressions of $\Delta_{S R, k}, Q_{k i}, \Phi_{k i}, \Delta_{R D, k}, R_{k i}, \phi_{k i}$. The equation (12) can be written as

$$
\gamma=\sqrt{\frac{P_{R}}{p_{u} N_{r}\left(\sum_{i=1}^{K} \alpha_{i}^{2}\left(N_{r}+\Delta_{S R, i}\right)+\sum_{i=1}^{K} \alpha_{i} \sum_{l \neq i}^{K} \alpha_{l} Q_{i l}\right)+\sigma_{R}{ }^{2} N_{r} \sum_{l=1}^{K} \alpha_{l}}}
$$

Proof: See Appendix B 


\subsection{Sum Rates in Three Different Scenarios}

In the actual communication process, the LOS path may not exist in the user to RS or RS to BS. Hence, in the following sections, three different models are deduced to deal with this situation. Case1) The effect of LOS path is considered merely in the users to the RS, Case2) The effect of LOS path is considered merely in the RS to the BS, and Case3) The effect of LOS path is out of consideration both in the users to the RS and the RS to the BS.

Case1: In this section, the Rician-factor between the RS and the BS is $-\infty \mathrm{dB}$, i.e., $\varepsilon_{i}=0(i=0,1, \ldots, K)$. Thus the RS to the BS can be regarded as the Rayleigh fading channel. i.e., $\Delta_{R D, k}=1, R_{k i}=1$, for $i=0,1, \ldots, K$. no LOS path between the RS and BS. The following theorem 2 is obtained.

Theorem 2: When the effect of LOS path exists merely in the users to the RS, the analytical exact sum rate of the uplink for Massive MIMO two-hop relay system is given by

$$
R_{\text {sum }, \text { case } 1}=\frac{1}{2} \sum_{k=1}^{K} \log _{2}\left(1+\frac{A_{\text {case } 1, k}}{B_{\text {case } 1, k}+C_{\text {case } 1, k}+D_{\text {case }, k}}\right)
$$

Similar to the calculations of equations (18), (19), (20), and (21), term $A_{\text {case } 1, k}, B_{\text {case } 1, k}$, $C_{\text {case } 1, k}$, and $D_{\text {case } 1, k}$ can be obtained as follows

$$
\begin{gathered}
A_{\text {case } 1, k}=\gamma^{2} p_{u} \alpha_{k} \beta_{k} N_{r} N_{d}\left[\alpha_{k} \beta_{k}\left(N_{r}+\Delta_{S R, k}\right)\left(N_{d}+1\right)+\sum_{i \neq k}^{K} \alpha_{i} \beta_{i} Q_{i k}\right] \\
B_{\text {case } 1, k}=\gamma^{2} p_{u} \beta_{k} N_{r} N_{d} \sum_{j \neq k}^{K} \alpha_{j}\left[\alpha_{k} \beta_{k} Q_{k j} N_{d}+\sum_{i \neq j}^{K} \alpha_{i} \beta_{i} Q_{i j}+\alpha_{j} \beta_{j}\left(N_{r}+\Delta_{S R, j}\right)\right] \\
C_{\text {case } 1, k}=\gamma^{2} \beta_{k} N_{r} N_{d} \sigma_{R}^{2}\left[\alpha_{k} \beta_{k} N_{d}+\sum_{i=1}^{K} \alpha_{i} \beta_{i}\right] \\
D_{\text {case } 1, k}=\sigma_{D}^{2} \beta_{k} N_{d}
\end{gathered}
$$

Case2: In this case, the Rician-factor between the user and the RS is also $-\infty \mathrm{dB}$, i.e., $\mu_{i}=0(i=0,1, \ldots, K)$. Thus, the user to the RS can be regarded as Rayleigh fading channel, i.e., $\Delta_{S R, k}=1, Q_{k i}=1$, for $i=0,1, \ldots, K$ no LOS path between the user and RS. The following theorem 3 is obtained.

Theorem 3: When the effect of LOS path exists merely in the RS to the BS, the analytical exact sum rate of the uplink for Massive MIMO two-hop relay system can be given by

$$
R_{\text {sum }, \text { case } 2}=\frac{1}{2} \sum_{k=1}^{K} \log _{2}\left(1+\frac{A_{\text {case } 2, k}}{B_{\text {case } 2, k}+C_{\text {case } 2, k}+D_{\text {case } 2, k}}\right)
$$

Similar to the calculations of equations (18), (19), (20), and (21), term $A_{\text {case } 2, k}, B_{\text {case } 2, k}$, $C_{\text {case } 2, k}$, and $D_{\text {case } 2, k}$ can be obtained as follows

$$
\begin{gathered}
A_{\text {case } 2, k}=\gamma^{2} p_{u} \alpha_{k} \beta_{k} N_{r} N_{d}\left[\alpha_{k} \beta_{k}\left(N_{r}+1\right)\left(N_{d}+\Delta_{R D, k}\right)+\sum_{i \neq k}^{K} \alpha_{i} \beta_{i} R_{k i}\right] \\
B_{\text {case } 2, k}=\gamma^{2} p_{u} \beta_{k} N_{r} N_{d} \sum_{j \neq k}^{K} \alpha_{j}\left[\alpha_{k} \beta_{k}\left(N_{d}+\Delta_{R D, k}\right)+\sum_{i \neq k}^{K} \alpha_{i} \beta_{i} R_{k i}+\alpha_{j} \beta_{j} R_{k j} N_{r}\right]
\end{gathered}
$$

$$
\begin{gathered}
C_{\text {case } 2, k}=\gamma^{2} \beta_{k} N_{r} N_{d} \sigma_{R}^{2}\left[\alpha_{k} \beta_{k}\left(N_{d}+\Delta_{R D, k}\right)+\sum_{i \neq k}^{K} \alpha_{i} \beta_{i} R_{k i}\right] \\
D_{\text {case } 2, k}=\sigma_{D}{ }^{2} \beta_{k} N_{d}
\end{gathered}
$$

Case3: In this case, the Rician-factor from the user to the RS and the RS to the BS are both $-\infty \mathrm{dB}$, i.e., $\varepsilon_{i}=0, \mu_{i}=0(i=0,1, \ldots, K), \Delta_{S R, k}=1, Q_{k i}=1, \Delta_{R D, k}=1, R_{k i}=1$. Therefore, 
the model degenerates into a Rayleigh channel model. And theorem 4 can be obtained as follows:

Theorem 4: When the effect of LOS path is out of consideration both in the user to the RS and the RS to the BS, the analytical exact sum rate of the uplink for Massive MIMO two-hop relay system can be given as:

$$
R_{\text {sum }, \text { case } 3}=\frac{1}{2} \sum_{k=1}^{K} \log _{2}\left(1+\frac{A_{\text {case } 3, k}}{B_{\text {case } 3, k}+C_{\text {case } 3, k}+D_{\text {case } 3, k}}\right)
$$

Similar to the calculations of equations (18), (19), (20), and (21), term $A_{\text {case } 3, k}, B_{\text {case }, k}$, $C_{\text {case }, k}$, and $D_{\text {case }, k}$ can be obtained as follows:

$$
\begin{gathered}
A_{\text {case } 3, k}=\gamma^{2} p_{u} \alpha_{k} \beta_{k} N_{r} N_{d}\left[\alpha_{k} \beta_{k}\left(N_{r}+1\right)\left(N_{d}+1\right)+\sum_{i \neq k}^{K} \alpha_{i} \beta_{i}\right] \\
B_{\text {case } 3, k}=\gamma^{2} p_{u} \beta_{k} N_{r} N_{d} \sum_{j \neq k}^{K} \alpha_{j}\left[\alpha_{k} \beta_{k} N_{d}+\sum_{i=1}^{K} \alpha_{i}+\alpha_{j} \beta_{j} N_{r}\right] \\
C_{\text {case }, k}=\gamma^{2} \beta_{k} N_{r} N_{d} \sigma_{R}{ }^{2}\left[\alpha_{k} \beta_{k} N_{d}+\sum_{i=1}^{K} \alpha_{i} \beta_{i}\right] \\
D_{\text {case } 3, k}=\sigma_{D}{ }^{2} \beta_{k} N_{d}
\end{gathered}
$$

\section{Numerical Results}

In this section, we evaluate the sum rate of the uplink achievable of the Massive MIMO two-hop relay system based on the proposed models. In order to verify the validity of the derivation of expressions in section 3, we visualize the performances of proposed models under different RS antennas and Rician-factors. Assume that the shortest distance from the user to the relay is $r_{\min }=10$, and $s_{k}$ is a log-normal random variable with standard derivation $8 \mathrm{~dB}$, and path loss exponent $v=3.8$. The value of $\alpha_{k}$ and $\beta_{k}$ can be calculated by $\alpha_{k}=s_{k}\left(r_{S R, k} / r_{\text {min }}\right)^{-v}$ and $\beta_{k}=s_{k}$. Through $\left[\mathbf{D}_{S R}\right]_{k k}=\alpha_{k}$ and $\left[\mathbf{D}_{R D}\right]_{k k}=\beta_{k}$ we can then obtain $\mathbf{D}_{S R}$ and $\mathbf{D}_{R D}$. The arrival angles $\theta_{S R, i}$ and $\theta_{R D, i}$ are uniformly distributed in the interval $\left[-\frac{\pi}{2}, \frac{\pi}{2}\right]$ and the variance ${\sigma_{R}}^{2}={\sigma_{D}}^{2}=1$.Finally, let $\mu_{k}=\varepsilon_{k}=10 \mathrm{db}$ and substitute $\mu_{k} 、 \varepsilon_{k} 、 \theta_{S R, i}$ and $\theta_{R D, i}$ into $\left[\boldsymbol{\Omega}_{S R}\right]_{k k}=\mu_{k}$, $\left[\boldsymbol{\Omega}_{R D}\right]_{k k}=\varepsilon_{k}, \Delta_{S R, k}, Q_{k i}, \Phi_{k i}, \Delta_{R D, k}, R_{k i}$ and $\phi_{k i}$ to get $\boldsymbol{\Omega}_{S R}, \boldsymbol{\Omega}_{R D}, \Delta_{S R, k}$, $Q_{k i}, \Phi_{k i}, \Delta_{R D, k}, R_{k i}$ and $\phi_{k i}$ parameters [7].

\subsection{Sum Rate Change with the Number of Antennas}

Fig. 2, Fig. 3, Fig. 4 and Fig. 5 denote the analytical exact results and the Monte Carlo simulation results of the uplink achievable sum rates for the above four different models. When Rician-factor takes $0 \mathrm{~dB}$ and $10 \mathrm{~dB}$, where $K=10$ and $p_{u}=5,10,15$. For the above four different communication models, the Monte Carlo simulation results match the analytical exact results well, which demonstrates the validity of the analytical exact closed-form expressions derived in (17), (24), (29) and (34). 


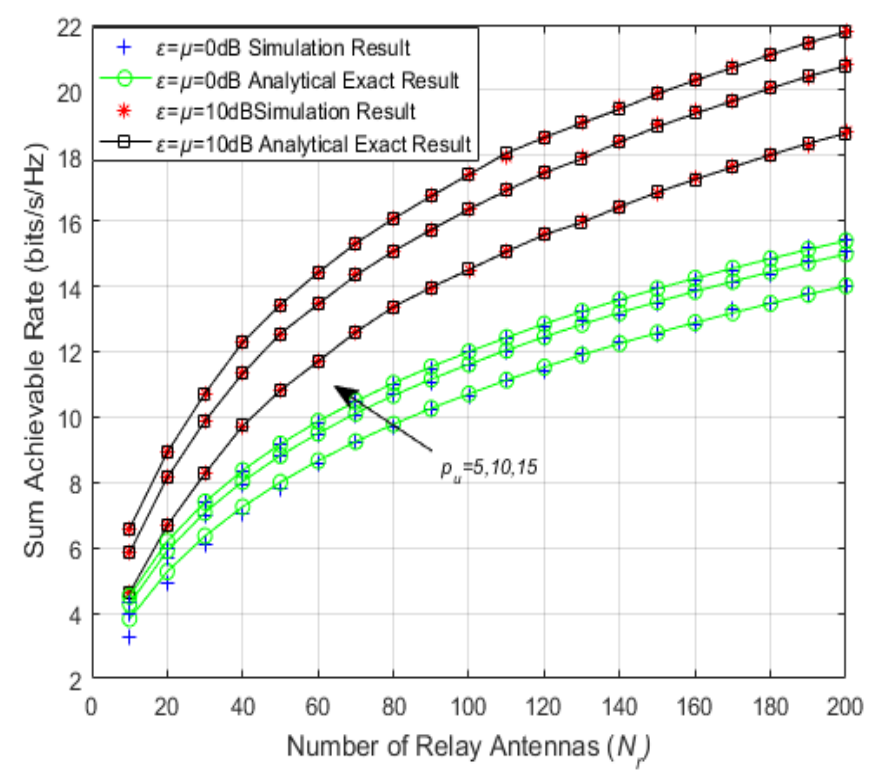

Fig. 2. the sum rate change with $N_{r}$ under both Rician fading channels

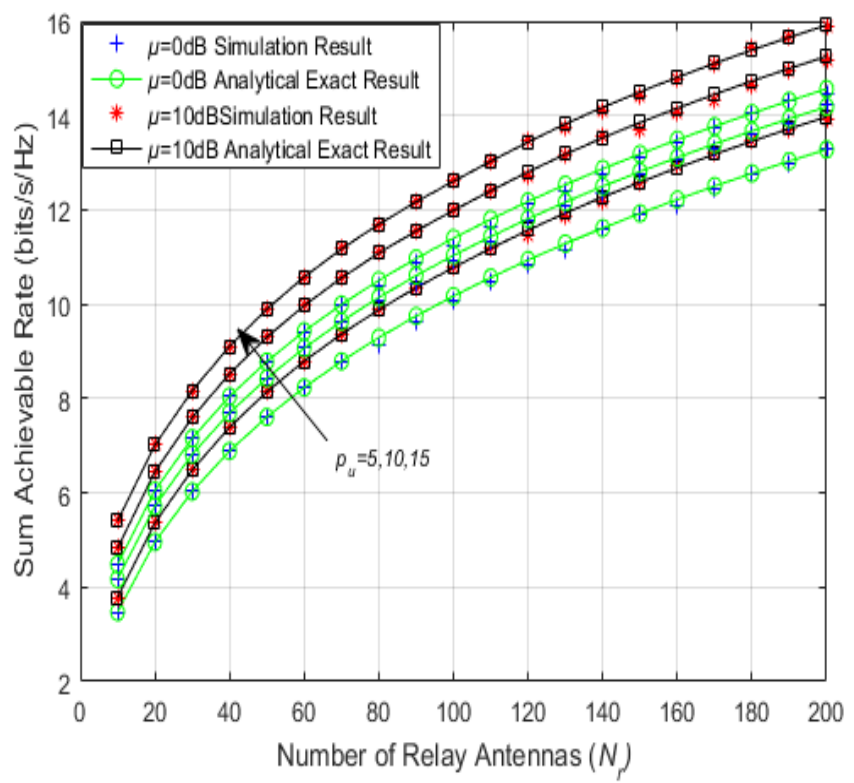

Fig. 3. Case1 the sum rate change with $N_{r}$ under the Rician fading channel exists between the user and the RS 


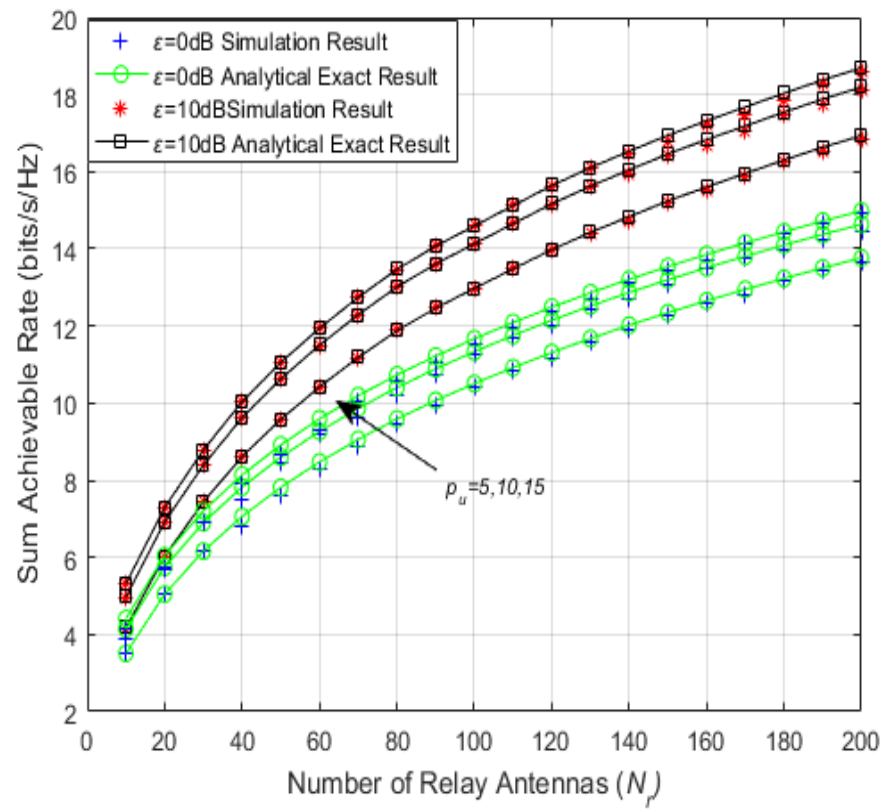

Fig. 4. Case2 the sum rate change with $N_{r}$ under the Rician fading channel exists between the RS and the BS

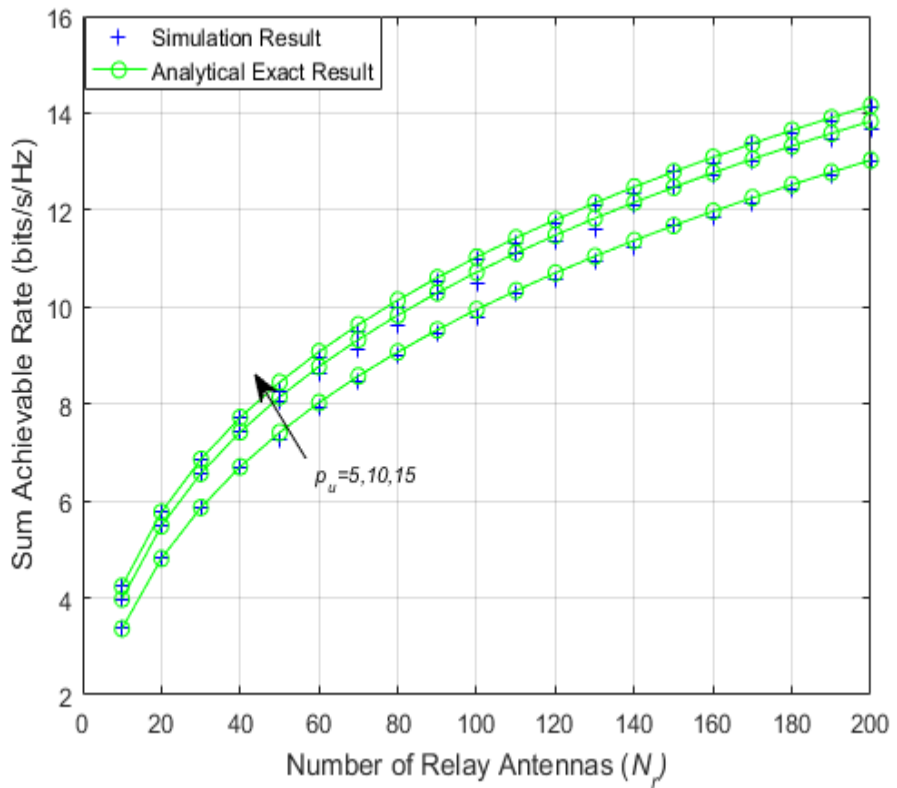

Fig. 5. Case3 the sum rate change with $N_{r}$ without Rician fading channel

Fig. 2, Fig. 3, Fig. 4 and Fig. 5 show that the sum rates increases as the $N_{r}$ increases, and conversely, the growth rate decreases as the $N_{r}$ increases. When the number of $N_{r}$ is large enough, the growth rate tends to zero, and the sum rate tends to a fixed value. What's 
more, Fig. 2, Fig. 3 and Fig. 4 indicate that the sum rate is positively correlated with Rician-factor when the transmission power is equal. Considering that the Rician-factor is fixed and the transmit power $p_{u}$ is increased by an equal interval, the sum rate increases much faster when the $p_{u}$ is small than when the $p_{u}$ is large. By comparing the four models, it can be easily found that the sum rate based on the Rician channel is larger than the sum rates in case 1 , case 2 , and case 3 , and the gap becomes larger with the increases of Rician-factor. Although there is only one LOS path in Fig. 3 and Fig. 4, the sum rate of case 3 is remarkably larger than case 2 . When the model degenerates into the Rayleigh model, the sum rate tends to lowest value. This indicates that the transmission rate can be increased when taking LOS path into consideration, which is consistent with the actual communication.

\subsection{Sum Rate Change with the Rician-Factor}

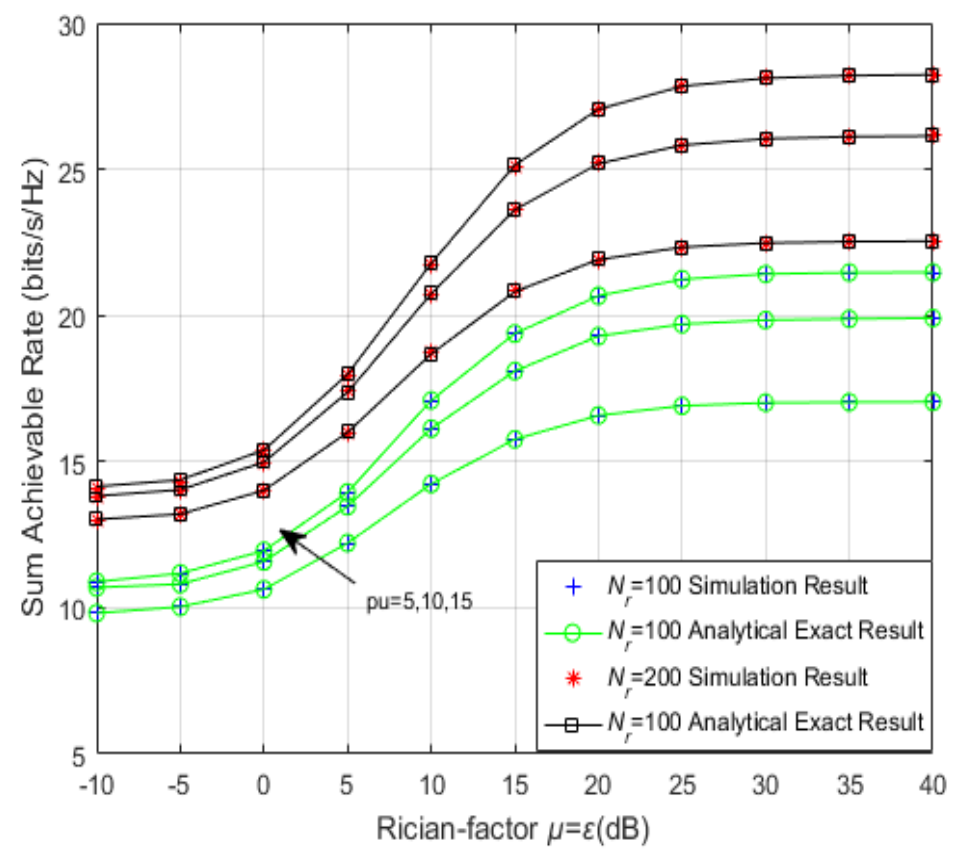

Fig. 6. the sum rate change with Rician-factor under both Rician fading channels.

Fig. 6 shows the variation of sum rate with the increase of Rician-factor. Specially, $N_{r}$ is set to 100 or 200 respectively, and $K=10, p_{u}=5,10$ and 15 . It can be learned from the above figure that the achievable sum rate is positively correlated with the Rician-factor, transmit power, and number of antennas. When the Rician-factor decreases to a relatively small value, the channel degenerates into a Rayleigh fading channel. And at around $25 \mathrm{~dB}$, the sum rate gets saturated and no longer varies with the growth of Rician-factor. 


\subsection{The Average Rate Change with the Number of Users}

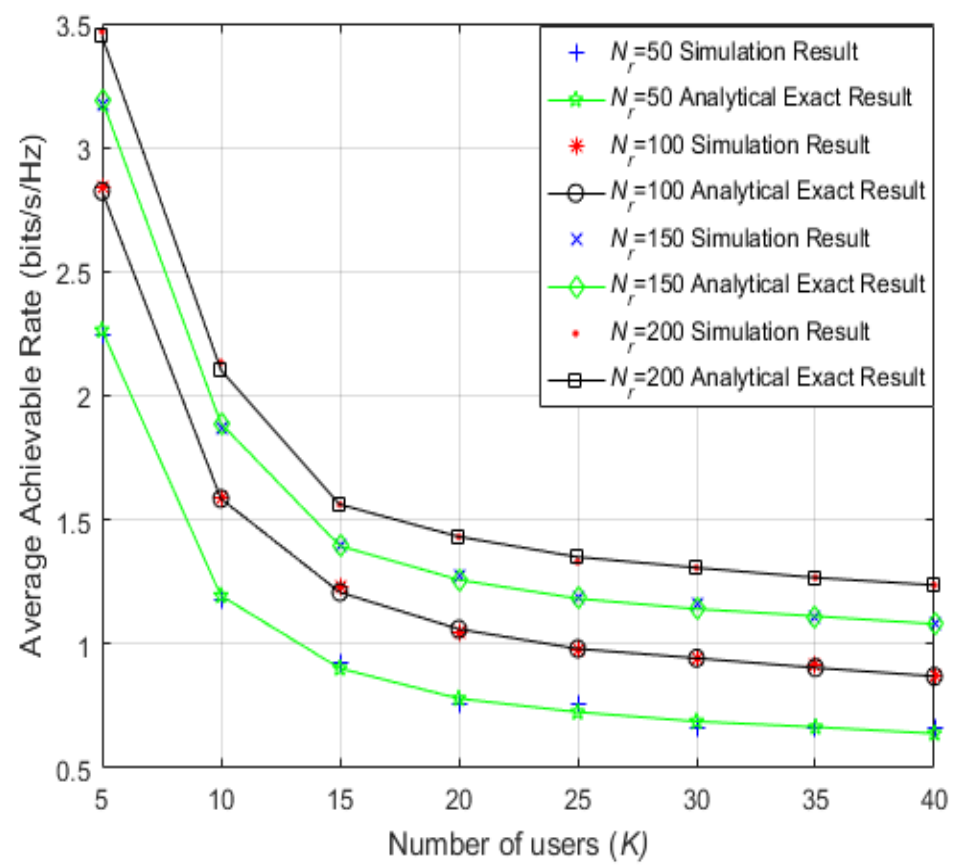

Fig. 7. Average rate under both Rician fading channels with perfect CSI versus $K$ for different $N_{r}$.

Fig. 7 shows the variation of average rate with the number of users. Specially, $N_{r}$ is 50, 100,150 , and 200 respectively, and $p_{u}=10, \mu_{i}=\varepsilon_{i}=10 \mathrm{~dB}$. Average per-user rate decreases logarithmically as the number of users increases. At the same time, the increase of $N_{r}$ improves the average per-user spectrum efficiency as well as the QoS of the system. Since the number of available antennas per user is increased, the degree of freedom is also increased and interference between multiple users can be better eliminated.

\section{Conclusions}

In order to make full use of the advantages of Massive MIMO two-hop relay technology, the performance of the uplink of the Massive MIMO two-hop relay system over Rician fading channel is studied in this paper. For perfect CSI, we derive the analytical exact closed-form expression of the uplink for the Massive MIMO two-hop relay system over Rician fading channels with $N_{r}$ and $N_{d}$ large enough. Then, we develop three the analytical exact closed-form expressions for sum rates of the uplink under three different scenarios to have a deep study of the effect of LOS path. Finally, simulation results show that the achievable sum rate of the Rician channel Massive MIMO two-hop relay system is significantly larger than the sum rates of the other three cases. Although case 2 and case 1 
only have Rician fading channel, the achievable sum rate of case 2 is larger than case1. When both channels degenerate into the Rayleigh fading channels, the achievable sum rate reach the minimum value in case 3 . The above results prove that the performance of communication system can be significantly improved when the LOS path is taken into account.

\section{Appendix A Proof of the Theorem1}

According to [21], we can get the following high-order statistics as:

$$
\begin{gathered}
\mathbb{E}\left\{\| \mathbf{g}_{S R, k}||^{2}\right\}=\alpha_{k} N_{r} \\
\mathbb{E}\left\{\| \mathbf{g}_{R D, k}||^{2}\right\}=\beta_{k} N_{d}
\end{gathered}
$$

From (17), the expectation of $\mathbb{E}\left\{\left|\mathbf{g}_{R D, k}^{H} \mathbf{G}_{R D} \mathbf{G}_{S R}^{H} \mathbf{g}_{S R, k}\right|^{2}\right\}$ is calculated as

$$
\begin{gathered}
\mathbb{E}\left\{\left|\mathbf{g}_{R D, k}^{H} \mathbf{G}_{R D} \mathbf{G}_{S R}^{H} \mathbf{g}_{S R, k}\right|^{2}\right\}=\mathbb{E}\left\{\left|\sum_{i=1}^{K} \mathbf{g}_{R D, k}^{H} \mathbf{g}_{R D, i} \mathbf{g}_{S R, i}^{H} \mathbf{g}_{S R, k}\right|^{2}\right\} \\
= \\
\sum_{i=1}^{K} \mathbb{E}\left\{\left|\mathbf{g}_{R D, k}^{H} \mathbf{g}_{R D, i} \mathbf{g}_{S R, i}^{H} \mathbf{g}_{S R, k}\right|^{2}\right\}+ \\
\sum_{i=1}^{K} \sum_{l \neq i}^{K} \underbrace{\mathbb{E}\left\{\mathbf{g}_{R D, k}^{H} \mathbf{g}_{R D, i} \mathbf{g}_{R D, l}^{H} \mathbf{g}_{R D, k}\right\} \mathbb{E}\left\{\mathbf{g}_{S R, i}^{H} \mathbf{g}_{S R, k} \mathbf{g}_{S R, k}^{H} \mathbf{g}_{S R, l}\right\}}_{=0} \\
\mathbb{E}\left\{\left|\mathbf{g}_{R D, k}^{H} \mathbf{g}_{R D, k}\right|^{2}\right\} \mathbb{E}\left\{\left|\mathbf{g}_{S R, k}^{H} \mathbf{g}_{S R, k}\right|^{2}\right\}+\sum_{i \neq k}^{K} \mathbb{E}\left\{\left|\mathbf{g}_{R D, k}^{H} \mathbf{g}_{R D, i}\right|^{2}\right\} \mathbb{E}\left\{\left|\mathbf{g}_{S R, i}^{H} \mathbf{g}_{S R, k}\right|^{2}\right\} \\
=\alpha_{k} \beta_{k} N_{r} N_{d}\left[\alpha_{k} \beta_{k}\left(N_{r}+\Delta_{S R, k}\right)\left(N_{d}+\Delta_{R D, k}\right)+\sum_{i \neq k}^{K} \alpha_{i} \beta_{i} Q_{i k} R_{k i}\right](43)
\end{gathered}
$$

Through (43) we can calculate the expected power of the signal $A_{k}$

$$
A_{k}=\gamma^{2} p_{u} \alpha_{k} \beta_{k} N_{r} N_{d}\left[\alpha_{k} \beta_{k}\left(N_{r}+\Delta_{S R, k}\right)\left(N_{d}+\Delta_{R D, k}\right)+\sum_{i \neq k}^{K} \alpha_{i} \beta_{i} Q_{i k} R_{k i}\right]
$$

Similarly, $\mathbb{E}\left\{\left|\mathbf{g}_{R D, k}^{H} \mathbf{G}_{R D} \mathbf{G}_{S R}^{H} \mathbf{g}_{S R, j}\right|^{2}\right\}$ in (17) can be calculated as

$$
\begin{gathered}
\mathbb{E}\left\{\left|\mathbf{g}_{R D, k}^{H} \mathbf{G}_{R D} \mathbf{G}_{S R}^{H} \mathbf{g}_{S R, j}\right|^{2}\right\} \\
= \\
\mathbb{E}\left\{\left|\mathbf{g}_{R D, k}^{H} \mathbf{g}_{R D, k} \mathbf{g}_{S R, k}^{H} \mathbf{g}_{S R, j}\right|^{2}\right\}+\sum_{\substack{i \neq k \\
i \neq j}}^{K} \mathbb{E}\left\{\left|\mathbf{g}_{R D, k}^{H} \mathbf{g}_{R D, i} \mathbf{g}_{S R, i}^{H} \mathbf{g}_{S R, j}\right|^{2}\right\}+ \\
\mathbb{E}\left\{\left|\mathbf{g}_{R D, k}^{H} \mathbf{g}_{R D, j} \mathbf{g}_{S R, j}^{H} \mathbf{g}_{S R, j}\right|^{2}\right\}
\end{gathered}
$$




$$
\begin{gathered}
=\mathbb{E}\left\{\left|\mathbf{g}_{R D, k}^{H} \mathbf{g}_{R D, k}\right|^{2}\right\} \mathbb{E}\left\{\left|\mathbf{g}_{S R, k}^{H} \mathbf{g}_{S R, j}\right|^{2}\right\}+\sum_{\substack{i \neq k \\
i \neq j}}^{K} \mathbb{E}\left\{\left|\mathbf{g}_{R D, k}^{H} \mathbf{g}_{R D, i}\right|^{2}\right\} \mathbb{E}\left\{\left|\mathbf{g}_{S R, i}^{H} \mathbf{g}_{S R, j}\right|^{2}\right\}+ \\
\mathbb{E}\left\{\left|\mathbf{g}_{R D, k}^{H} \mathbf{g}_{R D, j}\right|^{2}\right\} \mathbb{E}\left\{\left|\mathbf{g}_{S R, j}^{H} \mathbf{g}_{S R, j}\right|^{2}\right\} \\
=\alpha_{j} \beta_{k} N_{r} N_{d}\left[\alpha_{k} \beta_{k} Q_{k j}\left(N_{d}+\Delta_{R D, k}\right)+\sum_{i \neq k, i \neq j}^{K} \alpha_{i} \beta_{i} Q_{i j} R_{k i}+\alpha_{j} \beta_{j} R_{k j}\left(N_{r}+\Delta_{S R, j}\right)\right]
\end{gathered}
$$

Then the power of the interference $B_{k}$ is written as

$$
\begin{gathered}
B_{k}=\gamma^{2} p_{u} \beta_{k} N_{r} N_{d} \sum_{j \neq k}^{K} \alpha_{j}\left[\alpha_{k} \beta_{k} Q_{k j}\left(N_{d}+\Delta_{R D, k}\right)+\sum_{i \neq k, i \neq j}^{K} \alpha_{i} \beta_{i} Q_{i j} R_{k i}+\alpha_{j} \beta_{j} R_{k j}\left(N_{r}+\right.\right. \\
\left.\left.\Delta_{S R, j}\right)\right]
\end{gathered}
$$

Then we calculate $\mathbb{E}\left\{\left|\mathbf{g}_{R D, k}^{H} \mathbf{G}_{R D} \mathbf{G}_{S R}{ }^{H}\right|^{2}\right\}$ in (17) as

$$
\mathbb{E}\left\{\left|\mathbf{g}_{R D, k}^{H} \mathbf{G}_{R D} \mathbf{G}_{S R}^{H}\right|^{2}\right\}=\mathbb{E}\left\{\left(\sum_{i=1}^{K} \mathbf{g}_{R D, k}^{H} \mathbf{g}_{R D, i} \mathbf{g}_{S R, i}^{H}\right)\left(\sum_{l=1}^{K} \mathbf{g}_{S R, l} \mathbf{g}_{R D, l}^{H} \mathbf{g}_{R D, k}\right)\right\}
$$

The above formula is not zero only when $i=l$

$$
\begin{gathered}
\mathbb{E}\left\{\left|\mathbf{g}_{R D, k}^{H} \mathbf{G}_{R D} \mathbf{G}_{S R}^{H}\right|^{2}\right\} \\
=\mathbb{E}\left\{\left|\mathbf{g}_{R D, k}^{H} \mathbf{g}_{R D, k}\right|^{2}\right\} \mathbb{E}\left\{\left\|\mathbf{g}_{S R, k}\right\|^{2}\right\}+\mathbb{E}\left\{\sum_{i \neq k}^{K}\left|\mathbf{g}_{R D, k}^{H} \mathbf{g}_{R D, i}\right|^{2}\left\|\mathbf{g}_{S R, i}^{H}\right\|^{2}\right\} \\
=\mathbb{E}\left\{\left|\mathbf{g}_{R D, k}^{H} \mathbf{g}_{R D, k}\right|^{2}\right\} \mathbb{E}\left\{\left\|\mathbf{g}_{S R, k}\right\|^{2}\right\}+\sum_{i \neq k}^{K} \mathbb{E}\left\{\left|\mathbf{g}_{R D, k}^{H} \mathbf{g}_{R D, i}\right|^{2}\right\} \mathbb{E}\left\{\left\|\mathbf{g}_{S R, i}^{H}\right\|^{2}\right\}
\end{gathered}
$$

Then the effect of the AWGN at the RS is given by

$$
C_{k}=\gamma^{2} \beta_{k} N_{r} N_{d} \sigma_{R}^{2}\left[\alpha_{k} \beta_{k}\left(N_{d}+\Delta_{R D, k}\right)+\sum_{i \neq k}^{K} \alpha_{i} \beta_{i} R_{k i}\right] \text { (49) }
$$

Then the effect of the AWGN at the BS is given by

$$
\begin{gathered}
D_{k}=\sigma_{D}{ }^{2} \mathbb{E}\left\{\left\|\mathbf{g}_{R D, k}\right\|^{2}\right\}=\sigma_{D}{ }^{2} \beta_{k} N_{d}(50) \\
\text { Appendix B } \\
\text { Proof of Amplification Factor }
\end{gathered}
$$

From (23), the expectation of the $i$ th diagonal element of $\mathbf{G}_{S R}^{H} \mathbf{G}_{S R} \mathbf{G}_{S R}^{H} \mathbf{G}_{S R}$ is calculated as

$$
\begin{gathered}
\mathbb{E}\left\{\left[\mathbf{G}_{S R}^{H} \mathbf{G}_{S R} \mathbf{G}_{S R}^{H} \mathbf{G}_{S R}\right]_{i i}\right\}=\mathbb{E}\left\{\sum_{l=1}^{K}\left|\mathbf{g}_{S R, i}^{H} \mathbf{g}_{S R, l}\right|^{2}\right\}= \\
\mathbb{E}\left\{\left|\mathbf{g}_{S R, i}^{H} \mathbf{g}_{S R, i}\right|^{2}\right\}+\mathbb{E}\left\{\sum_{l \neq i}^{K}\left|\mathbf{g}_{S R, i}^{H} \mathbf{g}_{S R, l}\right|^{2}\right\}=\alpha_{i}^{2} N_{r}\left(N_{r}+\Delta_{S R, i}\right)+\alpha_{i} N_{r} \sum_{l \neq i}^{K} \alpha_{l} Q_{i l}
\end{gathered}
$$

Then we can get

$$
\begin{aligned}
& \operatorname{tr}\left(\mathbb{E}\left\{\mathbf{G}_{S R}^{H} \mathbf{G}_{S R} \mathbf{G}_{S R}^{H} \mathbf{G}_{S R}\right\}\right)=\sum_{i=1}^{K} \mathbb{E}\left\{\left[\mathbf{G}_{S R}^{H} \mathbf{G}_{S R} \mathbf{G}_{S R}^{H} \mathbf{G}_{S R}\right]_{i i}\right\} \\
& \quad=\left(N_{r} \sum_{i=1}^{K} \alpha_{i}{ }^{2}\left(N_{r}+\Delta_{S R, i}\right)+N_{r} \sum_{i=1}^{K} \alpha_{i} \sum_{l \neq i}^{K} \alpha_{l} Q_{i l}\right)
\end{aligned}
$$

$\operatorname{tr}\left(\mathbb{E}\left\{\mathbf{G}_{S R}^{H} \mathbf{G}_{S R}\right\}\right)$ is easy to obtain

$$
\operatorname{tr}\left(\mathbb{E}\left\{\mathbf{G}_{S R}^{H} \mathbf{G}_{S R}\right\}\right)=\sum_{l=1}^{K} \mathbb{E}\left\{\mathbf{g}_{S R, l}^{H} \mathbf{g}_{S R, l}\right\}=N_{r} \sum_{l=1}^{K} \alpha_{l}
$$

Finally, from (12), the expression of $\gamma$ is given by 


$$
\gamma=\sqrt{\frac{P_{R}}{p_{u} N_{r}\left(\sum_{i=1}^{K} \alpha_{i}{ }^{2}\left(N_{r}+\Delta_{S R, i}\right)+\sum_{i=1}^{K} \alpha_{i} \sum_{l \neq i}^{K} \alpha_{l} Q_{i l}\right)+\sigma_{R}{ }^{2} N_{r} \sum_{l=1}^{K} \alpha_{l}}}
$$

\section{References}

[1] S. Wu, C. X. Wang, H. Haas, E. H. M. Aggoune, M. M. Alwakeel, and B. Ai, “A non-stationary wideband channel model for massive MIMO communication systems,” IEEE Trans. Wireless Commun., vol. 14, no. 3, pp. 1434-1446, Mar, 2015. Article (CrossRef Link)

[2] H. Jiang, Z. C. Zhang, J. Dang, and L. Wu, “A Novel 3D Massive MIMO Channel Model for Vehicle-to-Vehicle Communication Environments,” IEEE Transactions on Communications. vol. 66, no. 1, pp. 79-90, Jan. 2018. Article (CrossRef Link)

[3] H. Jiang, Z. C. Zhang, and G. Gui, “Three-Dimensional Non-stationary Wideband Geometry-Based UAV Channel Model for A2G Communication Environments,” IEEE Access, vol. 7, pp. 26116-26122, Mar. 2019. Article (CrossRef Link)

[4] Q. Wang and Y. Jing, "Performance analysis and scaling law of MRC/MRT relaying with CSI error in multi-pair massive MIMO systems,” IEEE Trans. Wireless Commun., vol. 16, no. 9, pp. 5882-5896, Sept.2017. Article (CrossRef Link)

[5] X. Wang, Y. Wang, and R. Sun, "Approximate sum rate for massive multiple-input multiple-output two-way relay with Ricean fading,” IET Commun., vol. 10, no. 12, pp. 14931500, Aug. 2016. Article (CrossRef Link)

[6] P. Dong, H. Zhang, W. Xu, and X. You, "Efficient low-resolution ADC relaying for multiuser massive MIMO system,” IEEE Trans. Veh. Techonol., vol. 66, no. 12, pp. 11039-11056, Dec. 2017. Article (CrossRef Link)

[7] C. Kong, A. Mezghani, C. Zhong, A. L. Swindlehurst, and Z. Zhang, "Multipair massive MIMO relaying systems with one-bit ADCs and DACs,” IEEE Trans. Signal Process., vol. 66, no. 11, pp. 2984-2997, Jun. 2018. Article (CrossRef Link)

[8] C. Kong, C. Zhong, S. Jin, S. Yang, H. Lin, and Z. Zhang, "Full-duplex massive mimo relaying systems with low-resolution adcs,” IEEE Trans.Wireless Commun., vol. 16, no. 8, pp. 5033-5047, Aug. 2017. Article (CrossRef Link)

[9] J. Liu, J. Xu, W. Xu, S. Jin, and X. Dong, "Multiuser massive MIMO relaying with mixed-ADC receiver,” IEEE Signal Process. Lett., vol. 24, no. 1, pp. 76-80, Jan. 2017. Article (CrossRef Link)

[10] E. Larsson, O. Edfors, F. Tufvesson, and T. Marzetta, "Massive MIMO for next generation wireless systems,” IEEE Commun. Mag., vol. 52, no. 2, pp. 186-195, Feb. 2014. Article (CrossRef Link)

[11] L. Lu, G. Y. Li, A. L. Swindlehurst, A. Ashikhmin, and R. Zhang, “An overview of massive MIMO: Benefits and challenges,” IEEE J. Sel. Topics Signal Process., vol. 8, no. 5, pp. 742-758, Oct. 2014. Article (CrossRef Link) 
[12] H. Q. Ngo, E. G. Larsson, and T. L. Marzetta, "Energy and spectral effi- ciency of very large multiuser MIMO systems,” IEEE Trans. Commun., vol. 61, no. 4, pp. 1436-1449, Apr. 2013. Article (CrossRef Link)

[13] H. A. Suraweera, H. Q. Ngo, T. Q. Duong, C. Yuen, and E. G. Larsson, "Multi-pair amplify-and-forward relaying with very large antenna arrays," in Proc. of IEEE Int. Conf. Commun., Budapest, Hungary, pp. 4635-4640, Jun. 2013. Article (CrossRef Link)

[14] H. Cui, L. Song, and B. Jiao, "Multi-pair two-way amplify-and-forward relaying with very large number of relay antennas,” IEEE Trans. Wireless Commun., vol. 13, no. 5, pp. 2636-2645, May 2014. Article (CrossRef Link)

[15] S. Jin, X. Liang, K.-K. Wong, X. Gao, and Q. Zhu, "Ergodic rate analysis for multipair massive MIMO two-way relay networks,” IEEE Trans.Wireless Commun., vol. 14, no. 3, pp. 1480-1491, Mar. 2015. Article (CrossRef Link)

[16] T. L. Marzetta, "Noncooperative cellular wireless with unlimited numbers of base station antennas,” IEEE Trans. Wireless Commun., vol. 9, no. 11, pp. 3590-3600, Nov. 2010. Article (CrossRef Link)

[17] J. G. Proakis, Digital Communications, 4th ed., McGraw-Hill, New York, NY, USA, 2001. Article (CrossRef Link)

[18] S. Jin, X. Q. Gao, and X. H. You, "On the ergodic capacity of rank-1 Ricean fading MIMO channels,” IEEE Trans. Inf. Theory, vol. 53, no.2, pp. 502-517, Feb. 2007.

Article (CrossRef Link)

[19] G. Alfano, A. Lozano, A. M. Tulino, and S. Verdú, "Mutual information and eigenvalue distribution of MIMO Ricean channels” in Proc. of IEEE Int. Symp. Inf. Theory Appl. (ISITA), pp. 10-13, Oct. 2004. Article (CrossRef Link)

[20] N. Ravindran, N. Jindal, and H. C. Huang, "Beamforming with finite rate feedback for LOS MIMO downlink channels,” in Proc. of IEEE Global Commun. Conf. (GLOBECOM), pp. 42004204, Nov. 2007. Article (CrossRef Link)

[21] Q. Zhang, S. Jin, K.-K. Wong, H. Zhu, and M. Matthaiou, "Power scaling of uplink massive MIMO systems with arbitrary-rank channel means,” IEEE J. Sel. Topics Signal Process., vol. 8, no. 5, pp. 966-981, Oct. 2014. Article (CrossRef Link) 

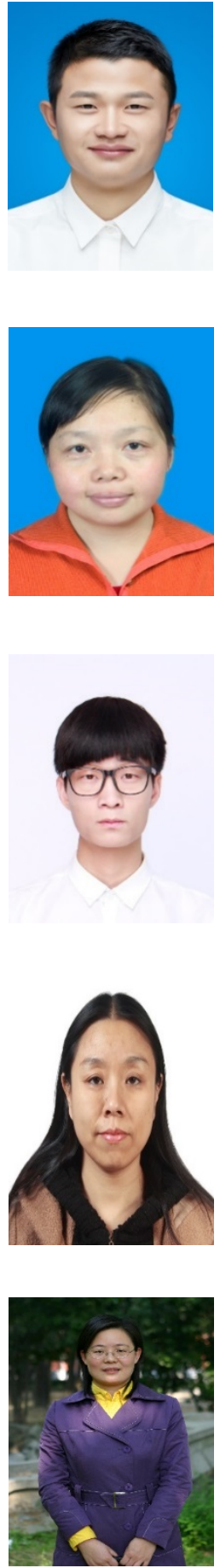

Zhang Yun received the M.S. and Ph.D. degrees from Nanjing Univ. of Posts \& Telecommunication, Nanjing, China, in 2005 and 2011, respectively. She has been a lectorate with the college of Electrical Science and Engineering, Nanjing Univ. of Posts \& Telecommunication since 2012. Her research interests are in the fields of adaptive signal processing, blind channel equalization, and digital and wireless communications.

Shengmei Zhao was born in 1968. She has been a Professor with School of Communication and Information Engineering of Nanjing Univ. of Posts \& Telecommunication, and a doctoral tutor. At present, At present, she mainly studies intelligent signal processing technologies in signals and channels in wireless networks, including cooperative communication and multi-radio technology, cooperative signals and information processing technologies. 